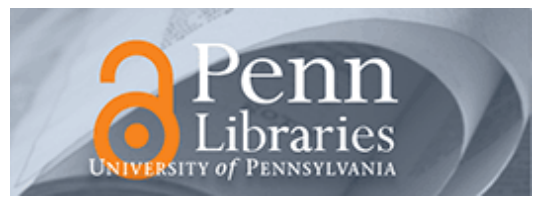

University of Pennsylvania

ScholarlyCommons

Finance Papers

Wharton Faculty Research

$9-2010$

\title{
Do Online Reviews Reflect a Product's True Perceived Quality? An Investigation of Online Movie Reviews Across Cultures
}

Noi Sian Koh

Nan Hu

Eric K. Clemons

University of Pennsylvania

Follow this and additional works at: https://repository.upenn.edu/fnce_papers

Part of the Finance and Financial Management Commons

\section{Recommended Citation}

Koh, N. S., Hu, N., \& Clemons, E. K. (2010). Do Online Reviews Reflect a Product's True Perceived Quality? An Investigation of Online Movie Reviews Across Cultures. Electronic Commerce Research and Applications, 9 (5), 374-385. http://dx.doi.org/10.1016/j.elerap.2010.04.001

This paper is posted at ScholarlyCommons. https://repository.upenn.edu/fnce_papers/95

For more information, please contact repository@pobox.upenn.edu. 


\title{
Do Online Reviews Reflect a Product's True Perceived Quality? An Investigation of Online Movie Reviews Across Cultures
}

\begin{abstract}
This paper investigates when the reported average of online ratings matches the perceived average assessment of the population as a whole, including the average assessments of both raters and nonraters. We apply behavioral theory to capture intentions in rating online movie reviews in two dissimilar countries - China and the United States. We argue that consumers' rating behaviors are affected by cultural influences and that they are influenced in predictable ways. Based on data collected from IMDB.com and Douban.com, we found significant differences across raters from these two different cultures. Additionally, we examined how cultural elements influence rating behavior for a hybrid culture Singapore. To study whether online consumer reviews are subjected to under-reporting bias, which is, consumers with extreme opinions are more likely to report their opinions than consumers with moderate reviews causing online reviews to be a biased estimator of a product's true quality, we compare the consumer reviews posted online with those from an experimental study. Our results shows that underreporting is more prevalent among US online network, thus online reviews are a better movie perceived quality proxy in China and Singapore than in the US.
\end{abstract}

\section{Keywords}

online reviews, movie ratings, cross-cultural comparisons, under-reporting bias

\section{Disciplines}

Business | Finance and Financial Management 


\section{Do Online Reviews Reflect a Product's True Perceived Quality? - An Investigation of Online Movie Reviews Across Cultures}

\author{
Noi Sian KOH \\ Singapore Management \\ University \\ noisian.koh.2006@smu.edu.sg
}

\author{
Nan HU \\ Singapore Management \\ University \\ hunan@smu.edu.sg
}

\author{
Eric K. CLEMONS \\ The Wharton School of the \\ University of Pennsylvania \\ clemons@,wharton.upenn.edu
}

\begin{abstract}
When does the reported average of online ratings match perceived average assessment? We apply behavioral theory to capture intentions in rating online movie reviews in two dissimilar countries - China and the United States. We argue that consumers' rating behaviors are affected by cultural influences. Based on data collected from imdb.com and douban.com, we found significant differences across raters from these two different cultures. Additionally, we examined how cultural elements influence rating behavior for a hybrid culture - Singapore. Under-reporting bias occurs when consumers with extreme opinions are more likely to report their opinions than consumers with moderate reviews, resulting in reviews that may be biased estimators of quality and certainly have higher variance. An experimental study shows that under-reporting is more prevalent among U.S. online social network, and thus that online reviews are more reflective of a movie's true perceived quality in Chinese and Singapore than in the U.S.
\end{abstract}

\section{Introduction}

User-generated online reviews are a major source of information and can reduce product uncertainty and help consumers infer product quality. Virtually all models for monetizing online social networks, from the well known like Facebook and YouTube to the more obscure like FirstWivesWorld, are based on trust and shared social values [11]. The most successful, like the relationship between TripAdvisor and Hotels.com, are based on trust; the greatest failures, like Facebook's Beacon, occur when this trust is violated [11]. Consumers do pay attention to what has been written in online social networks to make their purchase decisions [7, 8, 9, 10, 12, 25]. Prior research on consumer decision making has established that online reviews are considered more credible and trustworthy by consumers than traditional sources of information $[5,19]$. However, to what extent can the evaluations given by individuals in online networks be considered reliable and representative of the general consensus? This is crucial to understanding the prospects for monetizing social networks, and even to their continued relevance in marketing.

Prior research [18] has found evidence that online reviews may not be representative of the general consensus opinions due to under-reporting bias. Under-reporting bias is a form of self-selection bias described in literature on satisfaction [4]. Consumers who are very satisfied or dissatisfied will be more motivated to voice their opinions through reviews.

It has been found that under-reporting bias does exist in certain U.S. online review websites [18]. The reported average ratings (created by a small population) do not match the perceived average ratings of the general population. As consumers are increasingly dependent on online reviews to make purchase decisions, we study raters' behaviors to reveal whether under-reporting bias exists across cultures, and whether social network behavior will yield unbiased estimators of a product's quality.

As each review is an assessment of individuals' perceived product quality, this study first explores how such reports of perceived quality could be influenced by cultural factors. The behavior of individuals in online networks can be very different across cultures and may differ from offline behavior as well. Behavioral theory in social psychology asserts that specific salient beliefs influence behavioral intentions and subsequent behavior $[1,2$, 3]. Employing the constructs from behavioral theory - attitude, social norms and motivation - we seek to understand the following important questions which to the best of our knowledge have not been answered in the online review context: What factors motivate consumers to write online reviews? How does culture influence consumer behavior when writing reviews?

To identify the potential under-reporting bias that might render the mean of online movie reviews a biased estimator of movie quality, we examine the 
distribution of voluntarily posted online movie reviews, and compare that to the review distribution based on an offline experimental study, in which all respondents were asked to report their ratings for a number of movies that they have viewed and under what circumstances they are likely to write online reviews. Our results show that under-reporting bias varies across different cultures and online reviews more accurately reflect a movie's perceived quality in the Chinese social network than in the U.S. culture. Indeed, if social network behavior does not accurately reflect greater social norms in the U.S., adoption may prove more limited than currently expected.

The rest of the paper is organized as follows. The next section reviews related work. Section 3 describes the theoretical framework and research hypotheses. The research setting and methodology are presented in Section 4. Section 5 provides the analysis of our empirical findings. Section 6 discusses the limitations and concludes with suggestions for future research.

\section{Related Work}

\subsection{Attitude and Social Norms}

Taylor and Todd [28] describe a construct that behavioral beliefs influence attitudes which determine intentions and actual behavior. Behavioral beliefs arising from social pressure are termed normative beliefs [3], also termed social norms, i.e., the influence of a person's normative beliefs that others approve or disapprove a particular behavior. People's intentions to perform a particular action are influenced by social norms, or by their perception that important others think they ought to do so. In our context, social norms refer to the influence from consumers' normative belief that the behavior is accepted, encouraged and promoted by their social circle. Consumers may believe that their family, friends, and even online peers would favor certain online opinions, and this belief tends to influence their intentions and opinions. We examine how offline interactions and social norms influence online social network behavior.

\subsection{Cultural Differences}

Hofstede's [15] cultural dimensions serve as the most influential culture theory [22, 24]. His cultural framework has also received strong empirical support [26]. The framework was generated through the most extensive examination of cross-national values ever undertaken, involving 116,000 respondents from 40 countries. The results were consistent with the findings in 38 other studies [22]. Hofstede separated cultures on the basis of (a) masculinity-femininity, (b) individualism-collectivism, (c) power distance, (d) uncertainty avoidance, and the recent addition of the Confucian dimension of (e) long-term orientation [16]. We focus on (b) and (e).

Individualism-collectivism refers to the basic level of behavior regulation of either individuals or groups. Individualists view self and immediate family as relatively more important than the collective. Long-term orientation suggests following tradition, perseverance and practice of benevolence; short-term orientation on the other hand, is the tendency towards consumption and materialism. As these are longestablished and influential culture theories, we will be using these cultural constructs in our conceptual development to better understand how 'culture' manifests itself in online interactions [13].

\subsection{Under-Reporting Biases}

$\mathrm{Hu}$, Pavlou and Zhang [18] found evidence of two self-selection biases in the reporting of online consumer reviews that render mean ratings a biased estimator of product quality. Consumers with a favorable disposition towards a product will acquire the product and thus create more bias towards positive product reviews. Such a motivation to write product review is termed as acquisition bias. Secondly, consumers who are greatly dissatisfied or satisfied are more likely to report their review, compared to consumers with moderate sentiments. This is termed under-reporting bias. Based on the data collected from Amazon.com and an offline survey conducted on U.S. customers, they documented that while online consumer reviews have a U-shaped distribution, actual consumer assessments for the same set of products are normally distributed. They concluded that online reviews do not reflect a product's true perceived quality.

However, their study did not examine the raters' attitude and social norms across different culture, and thus under-reporting bias might vary across cultures. Therefore, we set out to understand raters' behavior across different cultures, - American and Chinese and to identify under what circumstances online reviews might reflect a product's true perceived quality. Since a large proportion of the movies watched by Chinese consumers were downloaded at no cost, acquisition bias is not very significant in our context and we focus on under-reporting bias.

The United States and China were chosen for this study because they represent almost reverse positions on several important cultural dimensions [15]. In addition, Singapore was chosen because of its mixture of Western and Eastern culture, which allows us to see how culture mediates the attitude and 
behavior in such a hybrid culture.

\section{Conceptual Development}

The proposed research model of online movie reviewers' behavior is adapted from Pavlou and Lin [24]. The dependent variable - online review behavior - captures consumers' reviewing intentions. Drawing from behavioral theory in social psychology, attitude towards the review and social norms directly influence online movie reviewing intentions $[3,23]$. These relationships are examined in terms of cultural differences on the dimensions of individualism-collectivism and long/short-term orientation. In addition, we looked at the motivation for consumers to write online reviews.

\subsection{Attitude}

Attitude has been proposed to influence behavioral intention in multiple theories such as Theory of Planned Behavior (TPB) [3] and the Theory of Reasoned Action (TRA) [14]. These theories have gained substantial empirical support $[20,24]$. Attitude here refers to an overall evaluation of the movie that an individual has viewed. A favorable attitude towards a movie will positively influence the rating of online movie reviews.

\subsection{Social Norms}

Social influence is related to Hofstede's dimension of individualism / collectivism. Collectivism refers to which individuals are integrated into groups and opinions are formed based on group norms [17]. Members of individualistic societies prefer freedom of expression, while those of collectivistic culture are more likely to seek to group consensus. As China is highly collectivist and United States is highly individualistic, we expect differences in the effect of societal influence on online movie reviewing.

Collectivist societies have strong relations within the extended family and acquaintances [17]. Their group relations focus on maintaining harmony by going along with the group's wishes and promoting long-term relationships [6]. We anticipate that members of a collectivist culture, such as China, would want to maintain harmonious writer-reader relationships in the online movie review website. On the other hand, members of individualist cultures, such as the U.S., do not generally feel as strong a need for the maintenance of group harmony and hence are free to openly express their appreciation or great dissatisfaction of the movies viewed.

Due to their colonial history, Singaporeans have been influenced by Western culture, but Eastern culture and values are also strong. Values such as obedience and harmony are important; and they value intense friendships and trust within the family. Therefore, we anticipate that Singapore is more of a collectivist society than the United States. Since China is highly collectivist and the U.S. is highly individualistic, we expect an attitudinal difference in the reviewing intentions between the two countries, as the following hypotheses propose.

H1: Collectivist societies focus on harmony and tend to write fewer bad reviews than individualist societies.

H2: Freedom of expression has a greater influence on reviews from individualist societies than from collectivists.

H3: Societal norms have a greater effect on online movie review in collectivist than in individualist societies.

According to Hofstede [16], China is extremely high on long-term orientation, which is a cultural dimension reflective of the teachings of Confucius. One of the key principles of Confucian teaching is the basic human benevolence toward others and this consists of treating others as one would like to be treated. We expect Chinese to be more generous in their ratings for Bottom-ranked movies.

H4: The ratings for Bottom-ranked movies given by collectivist societies will be less harsh and more generous than in individualist societies.

\subsection{Motivation}

Writing reviews seems to address basic human needs both for belonging to and gaining acceptance from groups in which they participate and for achieving status and recognition [21]. We hypothesize that consumers who participate in the writing of online reviews are motivated to meet these needs.

H5: The motivation to write movie review is affected by people's social needs to feel a sense of belonging and sharing.

H6: The motivation to write movie review is affected by people's needs for esteem and recognition.

Cultural differences may be at work here and we hope to gather sufficient responses in future to conduct further analysis on this part.

\section{Research Methodology}

To study the cultural differences in online movie review behavior, we gathered the reviews from two online movie review websites, i.e., IMDB.com and Douban.com. IMDB.com was chosen because it is the largest online movie review website with over 57 million visitors each month. For the Chinese website, we chose Douban.com because it is a cloned version of IMDB in China; and it is consistently ranked as 
one of the most popular online review website in China by Alexa Internet. ${ }^{1}$ To ensure we are comparing U.S. and Chinese cultures, we only extracted ratings given by U.S. reviewers in IMDB.

Our data sample is composed of two batches of data collection. For the first batch, reviews were collected on 1000 movies randomly selected from IMDB and 1000 movies randomly selected from Douban using a random counter on the movie identification number. By comparing the rating behavior of these 2000 movie items, we investigate the rating differences across these two websites. However, the conclusions might be influenced by the differences between the movies selected from these websites. To control this, we therefore conducted experiments on a second dataset. We first chose the Top 100 and Bottom 100 ranked movies in IMDB, then based on these movie titles, we selected the same movies in Douban.com. The reason for focusing on those Top and Bottom-ranked movies is that we believe it is more likely to observe underreporting bias for movies within such categories.

Table 1: Statistics of Dataset from IMDB.com

\begin{tabular}{|l|c|c|}
\hline Category & $\begin{array}{c}\text { Number } \\
\text { of Movies }\end{array}$ & Number of Ratings \\
\hline Top 100 & 100 & $2,944,037$ \\
\hline Bottom 100 & 100 & 191,411 \\
\hline Random 1000 & 1000 & 691,739 \\
\hline
\end{tabular}

Table 2: Statistics of Dataset from Douban.com

\begin{tabular}{|l|c|c|}
\hline Category & $\begin{array}{c}\text { Number } \\
\text { of Movies }\end{array}$ & Number of Ratings \\
\hline Top 100 & 100 & 483,680 \\
\hline Bottom 100 & 100 & 4,151 \\
\hline Random 1000 & 1000 & $14,645,654$ \\
\hline
\end{tabular}

The summary statistics ${ }^{2}$ of the dataset of the second batch from both websites are shown in Table 1 and 2. Both batches of data collection were gathered in December 2008. For each item, we collected the movie title, movie ID and review information. Specifically, for each movie review, we gathered the numeric rating, review date, helpful votes, total votes and the original text. On Douban, consumers can report an integer movie review on a 5point Likert-type scale, anchored at 1-star $=$ least satisfied and 5-star $=$ most satisfied. On IMDB,

\footnotetext{
1،"Top Sites China". Alexa Internet.

http://www.alexa.com/site/ds/top_sites?cc $=\mathrm{CN} \&$ ts_mode $=$ country \&lang=none. Retrieved on 5/10/2008.

2 The data analysis is based on data collected prior to $15 / 1 / 2009$. For robustness, we conducted the same analysis based on the data collected prior to Sept $15^{\text {th }}$ and Dec $15^{\text {th }}$ of 2008 , which rendered similar results
}

consumers can report an integer movie review on a 10-point Likert-type scale, anchored at 1 -star $=$ least satisfied and 10-star $=$ most satisfied.

\subsection{Experimental Measures}

After comparing the rating distribution of Chinese consumers to that of U.S. consumers, we conducted a survey in which respondents were asked to review the movies that they have viewed and we compared the results with those observed on IMDB and Douban. This results in less under-reporting bias.

Our respondents were university students attending business, information systems and economic courses. Each student was asked to review 16 movies that vary in terms of category and genre. For each movie, the subjects were asked to rate the movies they have viewed and report their intention and motivation to write online movie reviews. The online survey instrument was administered to 1,500 students. Our respondents are native Chinese speakers and Singaporeans who spoke Chinese fluently. Invitation emails explained the purpose of the study and requested participation. The respondents who clicked on the URL link provided in the email message were then directed to the online survey instrument. 87 Chinese students and 212 Singaporeans responded. Participation was voluntary and the response rate was approximately $19 \%$.

The movie rating scales for the survey were based on those of Douban.com. A preliminary version of the survey was generated and reviewed by doctoral students for clarity.

For U.S. users, we employed ratings from MovieLens $^{3}$ in place of our survey, due to insufficient survey response. Unlike IMDB, MovieLens is not an online review website but a recommender system. In MovieLens, users rate as many movies as possible (each user has to rate at least 35 movies) to improve the accuracy of the recommendations each will receive from the recommender system. Therefore, in MovieLens, the aim of the users for rating movies is to improve the quality of their own received recommendations and they are more likely to try to rate all the movies that they have watched. Hence, there is less under-reporting bias in MovieLens.

\section{Results}

We retrieved movie reviews of 1,000 randomly selected movie titles from each site, and we kept only

\footnotetext{
${ }^{3}$ This dataset is gathered for a GroupLens Research Project by the Department of Computer Science and Engineering at the University of Minnesota. It is publicly available for downloading at http://www.grouplens.org/taxonomy/term/14
} 
those movies with an average review of 3-stars in Douban and 5-stars in IMDB, the medians for the two sites. After that, we plotted the distribution of the ratings of Douban and IMDB respectively (as shown in Figure 1 and Figure 2). We expected to observe a normal distribution for both but did not.

Figures 1 and 2 show that for all movies with average review score equal to the median, (92 movies on IMDB and 151 on Douban) the rating histogram for IMDB is U-shaped, while for Douban is bell-shaped. Thus there are differences in the rating pattern even for average ranked movies. Preparing a review is more time-consuming than merely providing a numeric evaluation and might suggest a more serious effort to assess accurately. We compared the numeric ratings with and without textual reviews across IMDB and Douban and found them similar [29]. Our results still hold, with a bell-shaped rating histogram for Douban and a U-shaped rating histogram for IMDB.

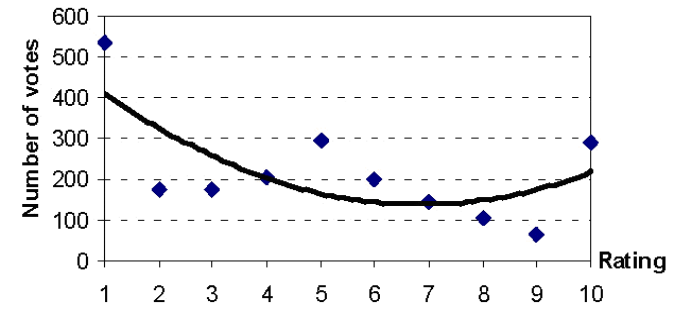

Figure 1: IMDB Movies with Average Rating $=5$ (92 out of 1000 movie items)

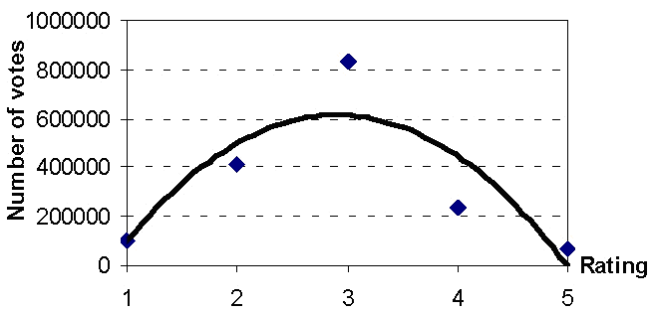

Figure 2: Douban Movies with Average Rating = 3 (151 out of 1000 movie items)

In the work that follows we compare individual rating behavior using the second dataset, in which we ensure that ratings are for the same set of movies, hence the observed differences in ratings are driven by the cultural differences, not the selection of movies in each sample. We focused on ratings of the most extreme movies, the Top-ranked and Bottomranked movies, in order to examine the most extreme rating behavior. Unless we state otherwise, all subsequent analysis was done using the second dataset.

Figures 3 and 4 show the rating distributions for the Top-ranked movies in IMDB and the corresponding rating distribution for the same set of movies in Douban. Figure 3 displayed a U-shaped distribution whereas Figure 4 has a unimodal distribution. It seems that most Chinese reviewers are reserved in giving full ratings as compared to U.S. reviewers. From both figures, we see that on the whole, the ratings for Top-ranked movies were not too different between both cultures. However, the mean rating difference between Douban and IMDB shows that Douban's mean ratings are significantly higher (pvalue $<0.001$ ), even though they are reserved in giving full ratings as compared to the U.S. reviewers.

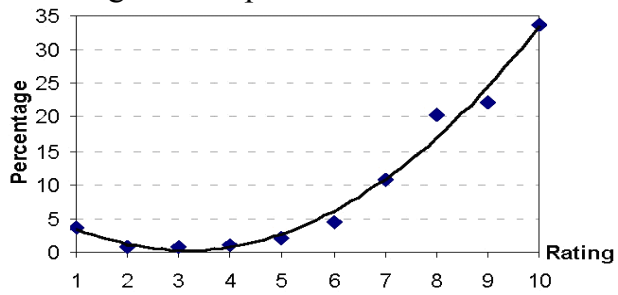

Figure 3: IMDB Top-ranked Movie Ratings

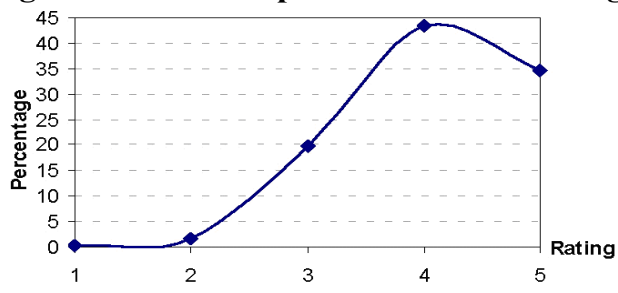

Figure 4: Corresponding (Top) Ratings in Douban

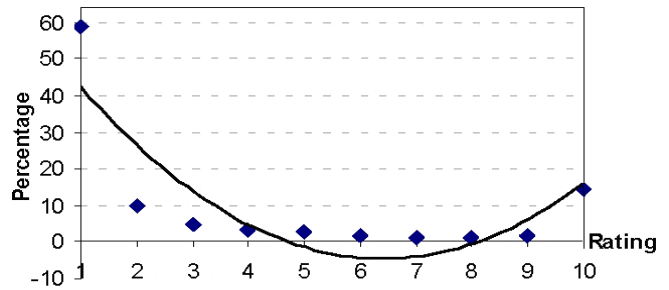

Figure 5: IMDB Bottom-ranked Movie Ratings

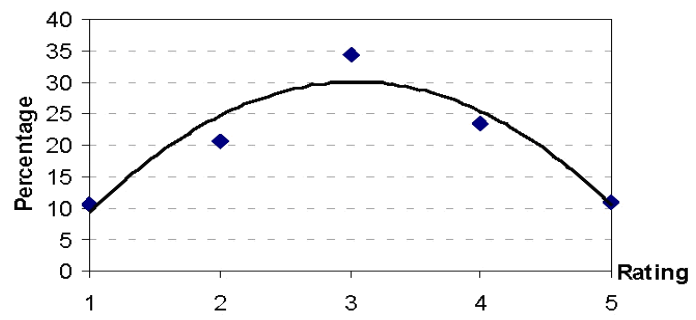

Figure 6: Corresponding Ratings in Douban

Figures 5 and 6 show the distribution of the ratings for the Bottom 100 movies in IMDB and the corresponding rating distribution for the same set of movies in Douban respectively. Unlike the results for the Top-ranked movies, there is a great difference between Chinese and American reviews for Bottomranked movies. Figure 5 shows that for the U.S. reviewers, most of the reviewers gave a very low rating of 1-star, resulting in a U-shaped distribution. For the Chinese reviewers, even when a movie is bad, the online reviews still demonstrate a bell-shaped distribution, as shown in Figure 6. This supports our 
hypothesis for $\mathrm{H} 4$, which argues that the ratings for bad movies given in collectivist societies will be more generous than individualist societies. U.S. reviewers on the other hand are more inclined to openly express their dissatisfaction (H2). This probably explains the difference in the number of reviews for the Bottom 100 movie category in IMDB $(191,411$ total ratings) and Douban (4,151 total ratings), providing support for $\mathrm{H} 1$.

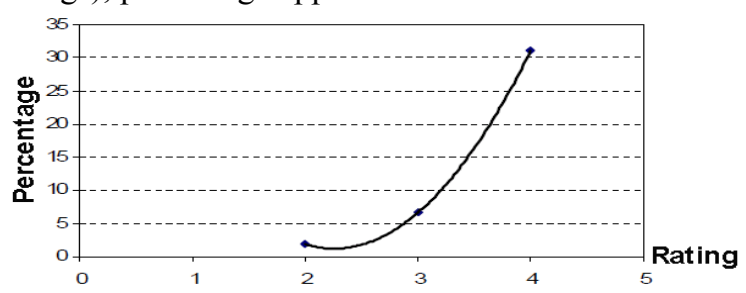

Figure 7: IMDB Top-ranked without Extremes

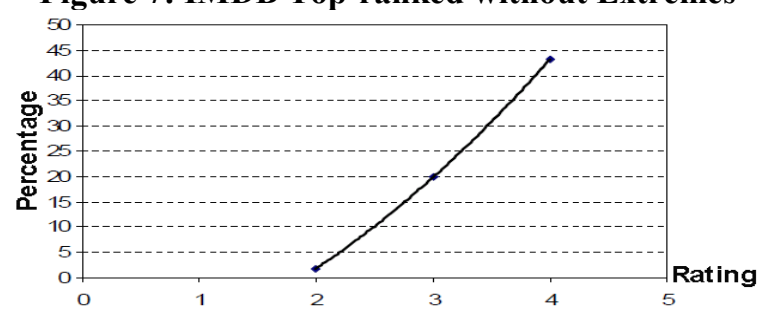

Figure 8: Corresponding without Extremes in Douban

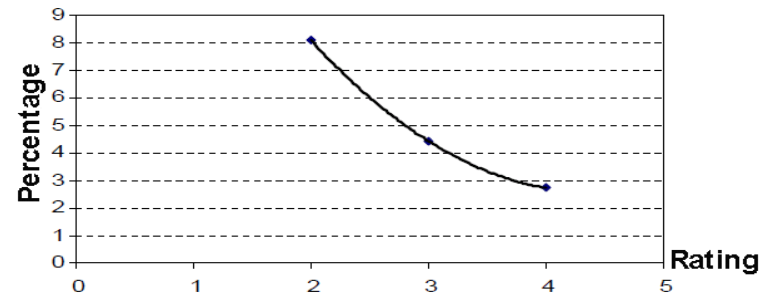

Figure 9: IMDB Bottom-ranked without Extremes

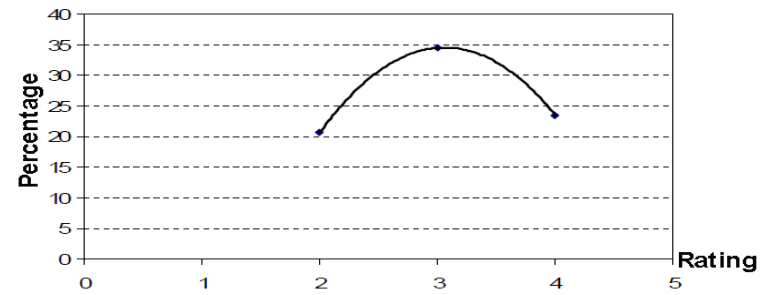

Figure 10: Corresponding without Extremes in Douban

For Figures 3 to 6 , if we remove the extreme ratings i.e., ratings $1,2,8$ and 10 for IMDB; ratings 1 and 5 for Douban, and standardize the scale to a 5 point Likert scale, it will result in Figures 7 to 10 respectively. The results suggest that the extremes are indeed over-represented in the American population relative to the Chinese population. There are several explanations. Perhaps Americans are more honest and willing to post extreme views because they are less influenced by the mean. Another explanation is that Americans might try to be different by giving extreme ratings, since simply giving an average rating does not show that they are individuals. The Chinese on the other hand are less open to giving extreme ratings either because they are more influenced by the consensus or they are less self-indulgent.

Overall there is a huge difference between consumer reviews of all the movies in IMDB and Douban, especially for the Bottom-ranked movies. It seems that American reviewers with extreme opinions are greatly over-represented relative to the population at large, which distorts American movie ratings relative to the true perceived quality. So far our results lead us to suspect that there is less underreporting bias amongst Chinese reviewers as shown by the bell-shaped curve in Figure 2. To address this concern, we conducted a survey to verify the existence of under-reporting bias, which will be discussed in Section 5.1.3.

\subsection{Hypotheses Testing}

5.1.1. Attitude and Social Norms on rating behavior.

The first experiment is to study how ratings are influenced by general consensus:

Rating $=f$ (Average rating, Average rating in $I M D B$ Bottom movies, Average rating in IMDB Top movies, Average rating in Douban Top movies, $t$ )

This is translated into the following empirical model:

$$
\begin{aligned}
\text { Rating }_{j t}= & \alpha_{1} * \text { AvgRating }_{j, t-1} \\
& +\alpha_{2} * \text { AvgRating }_{j, t-1} * \text { IMDB_Bottom } \\
& +\alpha_{3} * \text { AvgRating }_{j, t-1} * \text { IMDB_Top } \\
& +\alpha_{4} * \text { AvgRating }_{j, t-1} * \text { Douban_Top }_{-} \\
& +\alpha_{5} * t+\alpha_{6} * \text { Bottom_Dummy } \\
& +\alpha_{7} * \text { Imdb_Dummy }+\varepsilon_{j t}
\end{aligned}
$$

where:

\begin{tabular}{|l|l|}
\hline \multirow{2}{*}{$\begin{array}{l}\text { the sequence order of each } \\
\text { review to control the temporal } \\
\text { effect. The first review posted } \\
\text { for a movie will have } t=1 .\end{array}$} \\
\hline AvgRating $_{j, t-1}$ & $\begin{array}{l}\text { denotes the average consumer } \\
\text { rating at the time when the }(t-1)^{\text {th }} \\
\text { review was written for movie } \\
\text { item } j . \text { It captures the consensus } \\
\text { of consumers rating Bottom } \\
\text { movies in Douban. This is our } \\
\text { base group. }\end{array}$ \\
\hline AvgRating $_{j, t-1}$ & $\begin{array}{l}\text { IMDB_Bottom is a dummy } \\
\text { variable and equals 1 for the } \\
\text { ratings of Bottom movies in }\end{array}$ \\
\hline
\end{tabular}




\begin{tabular}{|l|l|}
\hline & IMDB. \\
\hline $\begin{array}{l}\text { AvgRating }{ }_{j, t-1} \\
\text { IMDB_Top }_{-}\end{array}$ & $\begin{array}{l}\text { IMDB_Top is a dummy variable } \\
\text { and equals 1 for the ratings of } \\
\text { Top movies in IMDB. }\end{array}$ \\
\hline AvgRating $_{j, t-1}$ & $\begin{array}{l}\text { Douban_Top is a dummy } \\
\text { variable 1 for the ratings of Top } \\
\text { movies in Douban. }\end{array}$ \\
\hline Bouban_Top & $\begin{array}{l}\text { denotes the dummy variable for } \\
\text { movies in the Bottom category }= \\
1 .\end{array}$ \\
\hline Imd _Dum_Dummy & $\begin{array}{l}\text { denotes the dummy variable for } \\
\text { movies in the IMDB category }= \\
1 .\end{array}$ \\
\hline
\end{tabular}

We estimated Model 1 using ordinary least square (OLS) to study how ratings are influenced by online review environment variables and reported the results in Table 3. We find that for both cultures, consumers are influenced by consensus. However, the level of influence is different for the Top and Bottom-ranked movies, particularly, where the parameter estimate for average rating is significantly positive (Para $=0.9279$ and P-Value $=0.0001$ ). This means that when Chinese consumers rate a bad movie, if the consensus rating is -1 , new consumers will give a rating of -0.9279 , a less negative score with tendency towards the center. The same principle applies when Chinese consumers rate a good movie. If the consensus rating is 1 , new consumers will give a rating of only about 0.24 , which is the sum of 0.9279 and 0.6917. Again, this is a less positive score with tendency towards the center. Overall, the results show that Chinese reviewers tend to keep within the boundary of the general consensus and in general will not give a more extreme rating than that which the online network has already given.

However, for the U.S. reviewers, the story is different, especially when they are facing a low quality movie. The interaction between IMDB_Bottom and average rating is positive (Para $=0.2631$ and P-Value $=0.0001)$. This indicates that when American consumers rate a bad movie, if the consensus rating is -1 , new consumers will give a rating of -1.19 , which is the sum of -0.9279 and 0.2631 , a more negative score with tendency over the boundary. Overall, it seems that U.S. reviewers are not confined to the rating boundary of the community, and they are more willing to post extreme reviews, especially when they are dissatisfied.
Table 3: Average ratings in IMDB and Douban

\begin{tabular}{|l|l|}
\hline Variable & Model \\
\hline Average rating & $0.9279^{* * *}$ \\
\hline Average rating*IMDB_Bottom & $0.2631^{+}$ \\
\hline Average rating*IMDB_Top & 0.1738 \\
\hline Average rating*Douban_Top & $-0.6917^{* * *}$ \\
\hline Intercept & $3.4503^{* * *}$ \\
\hline Sequence & $-0.0033^{* * *}$ \\
\hline IMDB Dummy & $1.6835^{* * *}$ \\
\hline Bottom Dummy & $-3.3391^{* * *}$ \\
\hline \multicolumn{2}{|c|}{ Legend: ${ }^{* * *} p<.001 ; * * p<.01 ;{ }^{*} p<.05 ;^{+} p<.10$}
\end{tabular}

\subsubsection{Motivation for Writing Online Reviews}

For this section, we examine what motivates individuals to post online movie reviews. In our survey, we asked the respondents:

(1) If they have been to movie review websites?

(2) Have they ever rated a movie in movie review websites?

(3) Under what circumstances will they rate a movie online - when they have extreme opinions, or when they want to share their opinions with others?

(4) How much influence does online movie reviews have on them?

(5) How often do they rate movies?

From the survey results collected, we perform a logistic regression to study the motivation for consumers to write online reviews. Our dependent variable is the frequency of respondents rating movies (Question 5), where 1 means 'never rated' and 5 represents 'often rated'. Our dependent variables capture the answers for question 1 to 4 :

Frequency $=f$ (been_to, rated, like, dislike, share, influence)

Table 4: Motivation to rate

\begin{tabular}{|l|l|}
\hline Variable & Model \\
\hline Been to movie review websites & $1.5663^{*}$ \\
\hline Have rated movies before & $1.1396^{* * *}$ \\
\hline Rate when like the movie & $0.6130^{*}$ \\
\hline Rate when dislike movie & 0.0604 \\
\hline $\begin{array}{l}\text { Want to share opinions with } \\
\text { others }\end{array}$ & $0.6647^{*}$ \\
\hline Online movie review's influence & 0.0331 \\
\hline Intercept & $-3.6553^{* * *}$ \\
\hline \multicolumn{2}{|l|}{ Legend: ${ }^{* * *} p<.001 ;{ }^{* *} p<.01 ;{ }^{*} p<.05{ }^{+} p<.10$} \\
\hline
\end{tabular}


In Table 4, the variables of "Been to movie review websites" and "Have rated movies before" are control variables, while the remaining are our variables of interest. We see that the "Want to share opinions with others" dominates all other needs (Para value $=$ 0.6647 and $\mathrm{P}$-value $<=0.05$ ) in motivating a consumer to write a review, giving strong support for $\mathrm{H} 5$. The next factor that motivates consumers to write online movie reviews is the need to express their liking for the movie (Parameter value $=-0.6130$ and $\mathrm{P}$ value $<=0.05$ ). Not surprisingly, when Chinese consumers are dissatisfied with a movie, they are not inclined to write online reviews. Finally, Chinese reviewers do not appear to write online reviews because of the need for esteem or to influence the views of others, rejecting our hypothesis for H6. To summarize, Chinese consumers are more likely to speak out when they like a movie instead of when they dislike a movie.

\subsubsection{Under-reporting Bias}

In the previous section, we documented that there are huge cultural influences affecting the behavior of Chinese and American customers when they post online movie reviews. In this section, we study whether such behavioral differences affect the accuracy of reviews as a quality indicator. Given that prior research [18] has found the existence of underreporting bias in United States consumers, causing online reviews to be a biased estimator of books' perceived quality, we study whether this is also true for movies and across different cultures.

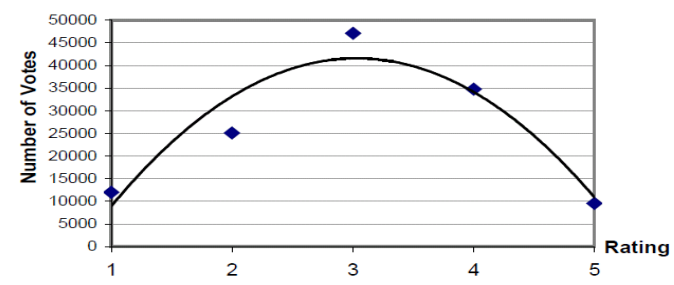

Figure 11: MovieLens Rating Distribution

For the comparison of U.S. movie ratings, we had to employ ratings from MovieLens. In MovieLens, users had to rate as many movies as possible (each user has to rate at least 35 movies) to improve the accuracy of the recommendations they will receive from the recommender system. We obtained a publicly available dataset, which contains 1,000,209 movie ratings for 3,900 movies contributed by reviewers to MovieLens. Since the aim of users in MovieLens is to improve the quality of their received recommendations, they are more likely to rate all the movies that they have watched and to rate them in a manner consistent with their own beliefs. Thus, we would expect much less reporting bias inside
MovieLens. And indeed from Figure 11, the results of the ratings given by the respondents in MovieLens display a unimodal distribution curve. In contrast, the online review results we obtained for IMDB is Ushaped, as shown in Figure 3 and 5. This confirmed and verified the existence of under-reporting bias in American consumers for movie reviews in IMDB. Such reporting bias causes online reviews to be a biased estimator of a movie's true perceived quality.

Next, we verify whether such under-reporting bias exists in another culture, in this case Chinese online network in Douban. The existence of underreporting bias is verified by a set of controlled experiments in which all respondents were asked to report their ratings for several movies that they have viewed. Figures 12 and 13 show the survey results by the Chinese students for the Top-ranked and Bottomranked movies respectively. The experiment revealed results similar to those gathered from the online website, Douban.com. In particular, the results obtained from Douban (Figure 6) and the results from our survey (Figure 13) follow a unimodal, almost normal distribution for the Bottom-ranked movies. By comparing the movie ratings in Douban for the Top-ranked movies (Figure 4) with the results from the survey (Figure 12), we can draw the similar conclusion that rating distributions are similar across these two channels for Bottom movie as well. Furthermore, the mean difference between online ratings and offline ratings is insignificant (tvalue $=0.45$ and $p$-value $=0.6569$ ). Our interpretation for this is that there is less under-reporting bias for Chinese online raters, and the overall online Chinese consumer opinion is a well representation of a movie's true perceived quality.

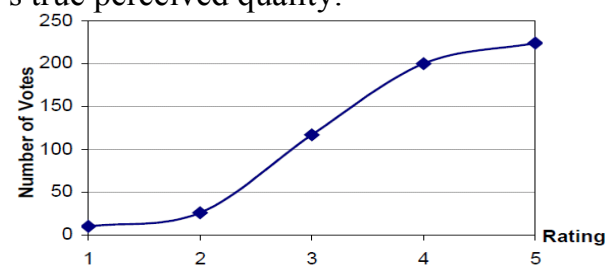

Figure 12: Chinese Students Survey for Topranked Movies

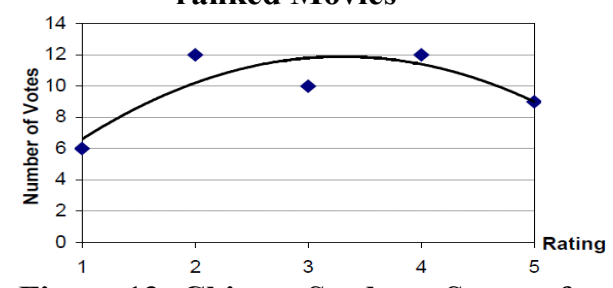

Figure 13: Chinese Students Survey for Bottom-ranked Movies

We have shown that the extent of under-reporting bias varies across cultures. In comparison to the results obtained from the United States reviewers, the 
existence of under-reporting bias is less severe among Chinese movie reviewers. Hence, the average ratings from Douban appear to be similar to those of the "silent consumers" who did not provide their ratings. However, in the case of IMDB, the average ratings may not be consistent with that of the "silent consumers" in the United States.

Most of the findings in this study are in accordance with expectations. As hypothesized, the difference in attitude had varying effects on online reviewing intentions for the collectivists and individualists. An explanation could lie in the influence of individualism in which individualists perceive that they are relatively free to follow their own wishes and outwardly express them. The fact that the number of reviews in the U.S. sample is much higher than that in the Chinese sample strengthens this assertion.

In terms of societal norms, collectivists display a much stronger adherence to the online network community's consensus. For the Chinese reviewers, there is concern for reconciliation, harmony and balance. Individualists would be expected to exhibit less concern for harmony and group norms.

To test if the results based on Chinese reviewers hold for other collectivist societies, we replicated our study in Singapore, which has been found [16] to be more collectivist than individualist due to its Confucian heritage in the majority of the population.

\subsubsection{Singapore Case Study}

In this section, we examine how cultural elements influence the attitudes and intentions in the hybrid culture of Singapore. Although three-fourths of the population is Chinese, Singaporeans undergo a British system of education, with English being the main medium. Due to their colonial history, Singaporeans have been influenced by Western culture, but Eastern culture and values are also strong. Obedience, harmony and concern for reconciliation are important. Cultural factors have been shown to be mediators of attitude and behavior in Singapore [27].

While Singaporean students are generally more exposed to Western values than their parents were, they still possess traditional Chinese values. This perhaps explains why the results for the Top-ranked movies (Figure 14) in the survey of Singaporean students were similar to those in Douban (Figure 4). In particular for the Bottom-ranked movies in Figure 15, the experimental results revealed unimodal (approximately normal) distribution with mostly moderate reviews, which is similar to the result for the Bottom 100 Movies in Douban.com, as in Figure 6.

$80.5 \%$ of our survey participants have been to movie review websites, but, only $14.2 \%$ have posted ratings. When asked when they would be most likely to provide movie review ratings, $66.5 \%$ responded that they would if they liked the movie very much and only $29.1 \%$ responded that they would if they were very disappointed. This suggests that reviewers in a collectivist culture are less likely to express their dissatisfaction, supporting $\mathrm{H} 1$.

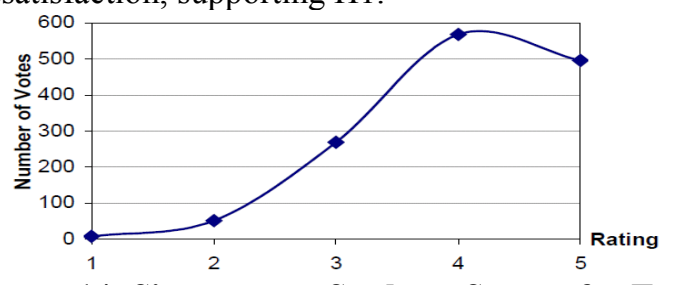

Figure 14: Singaporean Students Survey for Topranked Movies

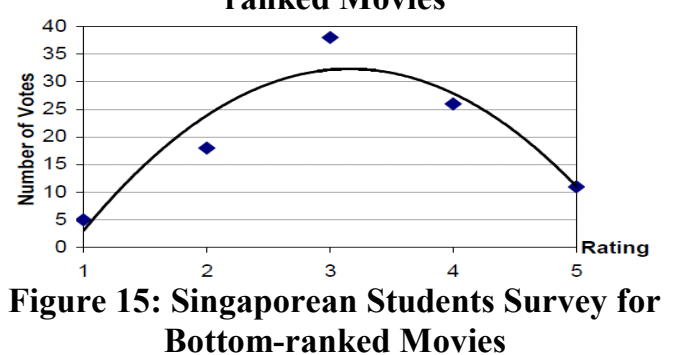

\section{Implications for Theory and Research}

This study contributes to our understanding of behavior when writing online movie reviews and, we believe, of the role of social norms in social networks more generally. A main contribution is the specification, justification and empirical validation of underreporting bias across cultures and a set of important cultural factors that associate with online review intentions. The results of this study validate the application of cultural dimensions theory proposed by Hofstede's [16] in explaining online movie reviewers' behavioral difference across cultures.

Based on the empirical study conducted over three different population groups, China, Singapore and the United States, we find that under-reporting bias varies across cultures and cultural differences play a significant role in online reviewing behavior.

Movies have always been made for their home markets, but with a hope for more general appeal. This study indicates that online social network reviewing behavior differs greatly from market to market, and might indeed lead a film's distributor to misjudge the size of a potential market abroad. In particular, a distributor might over-estimate the market in Singapore or in China, given the greater tolerance of reviewers, or, conversely, might underestimate the market in America given the viciousness of some American reviews. If online behavior is not representative of offline behavior, then information in online networks needs to be interpreted carefully 
before these reviews can be of use to either the community or marketers.

Finally, there are several limitations of this study. First, the study should be replicated with a nonstudent population. Second, future research could examine text and not just numerical reviews for a more comprehensive analysis. Third, it might be useful to see how results are similar in retail websites such as Amazon, eBay and others. Finally, further research on the behavior of Americans and of Chinese in online social networks would help to clarify the results observed.

\section{References}

[1] Ajzen, I., "From Intentions to Actions: A Theory of Planned Behavior," in Kuhl, J. and J. Beckmann (eds.), Action control: From cognition to behavior, 1985.

[2] Ajzen, I., Attitudes, Personality, and Behavior, The Dorsey Press, Chicago, Illinois, 1988.

[3] Ajzen, I., "The Theory of Planned Behavior," Organizational Behavior and Human Decision Processes, vol. 50, pp.179-211, 1991.

[4] Anderson, E. W. "Customer Satisfaction and Word of Mouth," Journal of Service Research, 1, 1, 5-17, 1998

[5] Bickart, B. and Schindler, R.M. "Internet forums as influential sources of consumer information". Journal of Marketing vol.15, no.3, pp.31-40, 2001.

[6] Bond, R. and Smith P.B., "Cross-Cultural Social and Organizational Psychology," Annual Review of Psychology, vol. 47, pp. 205-235, 1996.

[7] Chatterjee, P., "Online Reviews: Do Consumers Use Them?" Advances in Consumer Research, 28, 1, 129133, 2001

[8] Chevalier, J. and Mayzlin, D., "The Effect of Word of Mouth on Sales: Online Book Reviews," Journal of Marketing Research, 43, 3, 2006.

[9] Clemons, E. K. “How Information Changes Consumer Behavior and How Consumer Behavior Determines Corporate Strategy," in JMIS, vol. 25, no. 2 (Fall 2008), pp. 13 - 40.

[10] Clemons, E.K. and Gao, G., "Consumer informedness and diverse consumer purchasing behaviors: Traditional mass-market, trading down, and trading out into the long tail," Electronic Commerce Research and Applications, vol. 7, issue 1, pp. 3-17, 2008.

[11] Clemons, E.K., Barnett, S. and Appadurai A., "The Future of Advertising and the Value of Social Network Websites: Some Preliminary Examinations," in the Proceedings of the $9^{\text {th }}$ International Conference on Electronic Commerce, vol. 258, pp. 267-276, 2007.

[12] Dellarocas, C. The Digitization of Word-of-Mouth: Promise and Challenges of Online Feedback Mechanisms. Management Science, 49, 10, pp. 14071424, 2003

[13] Ess, C. and Sudweeks, F. Culture and computer- mediated communication: Toward new understandings, Journal of Computer-Mediated Communication, vol. 11, no 1, article 9, 2005.

[14] Fishbein, M. and Ajzen I., Belief, Attitude, Intention and Behavior: An Introduction to Theory and Research, Addison-Wesley, Reading, MA, 1975.

[15] Hofstede, G., Culture's Consequences, Sage, 1980.

[16] Hofstede, G., Culture's Consequences, Sage, 2001.

[17] Hofstede, G. and Bond M.H., "The Confucius Connection: From Cultural Roots to Economic Growth," Organizational Dynamics, 16, 4, 4-21, 1988.

[18] Hu, N., Pavlou, P.A. and Zhang J., "Can online reviews reveal a product's true quality?: empirical findings and analytical modeling of online word-of-mouth communication". Proc. of the 7th ACM conference on Electronic commerce, pp. 324 - 330, 2006.

[19] Li, C. and Bernoff J., Groundswell: Winning in a World Transformed by Social Technologies, 2008.

[20] Madden, T.J., Ellen P.S., and Ajzen I., “A Comparison of the Theory of Planned Behavior and the Theory of Reasoned Action," Personality and Social Psychology Bulletin, vol. 18, no. 1:3-9, 1992.

[21] Maslow A.H., “A Theory of Human Motivation”, Psychological Review, vol. 50, no.4, pp. 370-96, 1943.

[22] Nakata, C. and. Sivakumar K, "Instituting the Marketing Concept in a Multinational Setting: The Role of National Culture," Journal of the Academy of Marketing Science, vol. 29, no. 3:255-275, 2001.

[23] Pavlou, P.A., "What Drives Electronic Commerce? A Theory of Planned Behavior Perspective," Best Paper Proceedings of the Academy of Management Conference, Denver, CO, August 2002.

[24] Pavlou, P.A. and Lin C., "What Drives Electronic Commerce Across Cultures? A Cross-Cultural Empirical Investigation of the Theory of Planned Behavior", Journal of Electronic Commerce Research, vol. 3, no. 4, pp.240-253, 2002.

[25] Senecal, S. and Nantel, J., "The Influence of Online Product Recommendations on Consumers' Online Choices," Journal of Retailing, 80, 159-169, 2004.

[26] Sondergaard, M., "Research Note: Hofestede's Consequences: A Study of Reviews, Citations and Replications," Organization Studies, 15, 3, pp. 447456, 1994.

[27] Tan, C T and Farley J U, "The Impact of Cultural Patterns on Cognition and Intention in Singapore," The Journal of Consumer Research, 13, 4, pp.540544, 1987.

[28] Taylor, S. and Todd P. A., "Understanding Information Technology Usage: A Test of Competing Models," Information Systems Research, 6, 3, pp. 144176, 1995.

[29] Wu, F. and Huberman B., "Novelty and Collective Attention," in Proc. of the National. Academy Science, vol. 104, no. 45, pp.17599-17601, 2007. 